\title{
Effect of Verbal Empathy and Touch on Anxiety Relief in Patients Undergoing Flexible Bronchoscopy: Can Empathy Reduce Patients' Anxiety?
}

\author{
Sun Mi Choi Jinwoo Lee Young Sik Park Chang-Hoon Lee Sang-Min Lee \\ Jae-Joon Yim \\ Division of Pulmonary and Critical Care Medicine, Department of Internal Medicine, Seoul National University \\ College of Medicine, Seoul, Republic of Korea
}

\section{Key Words \\ Bronchoscopy · Anxiety · Empathy · Touch}

\begin{abstract}
Background: Patients undergoing flexible bronchoscopy usually experience anxiety before and during the procedure. Objectives: We performed this study to investigate whether verbal empathy and intentional touch from a bronchoscopist could reduce anxiety in patients undergoing flexible bronchoscopy. Methods: We conducted a prospective randomized trial in a university-affiliated hospital. Participants were randomly assigned to one of the following groups: a control group, a verbal empathy group, or a verbal empathy and touch group. The staff pulmonologist performing bronchoscopy expressed verbal empathy and used touch by speaking to the patient (using predefined short statements) and making eye contact, and by laying his/her left hand on the patient's right shoulder. We assessed the level of patient anxiety with a visual analog scale before and after the intervention. Results: Participants were 267 patients with a median age of 65 years; $62.2 \%$ were men. Although there were no differences in changes in anxiety between the three groups, subgroup analysis of the visual an-
\end{abstract}

alog scale including participants with higher baseline anxiety (empathy and touch group participants with anxiety $\geq 60 \mathrm{~mm}$; empathy group and empathy and touch group participants with anxiety $\geq 70 \mathrm{~mm}$ ) showed a larger reduction in anxiety than the control group. Conclusions: Verbal empathy and touch given by a bronchoscopist before bronchoscopy reduced anxiety in patients with high baseline anxiety levels.

c 2016 S. Karger AG, Basel

\section{Introduction}

Patients undergoing medical tests or procedures usually experience anxiety caused by both the tests themselves and fear of the test results [1]. For patients, anxiety associated with procedures may constitute an obstacle to the seeking of proper medical care. For physicians, patient anxiety could form a barrier to the performance of successful medical examinations [2, 3].

Flexible bronchoscopy (FB) is an essential tool for the diagnosis and treatment of various pulmonary conditions [4] and is one of the most common procedures performed by pulmonologists. With the induction of sedatives and

\section{KARGER}

E-Mail karger@karger.com

www.karger.com/res
(C) 2016 S. Karger AG, Basel

0025-7931/16/0926-0380\$39.50/0
Jae-Joon Yim, MD, PhD

Division of Pulmonary and Critical Care Medicine

Department of Internal Medicine, Seoul National University College of Medicine

101 Daehak-Ro, Jongno-Gu, Seoul 03080 (Republic of Korea)

E-Mail yimjj@snu.ac.kr 
Fig. 1. Flow diagram of the study. * Endobronchial biopsy, bronchial washing, brushing, bronchoalveolar lavage, or transbronchial lung biopsy.

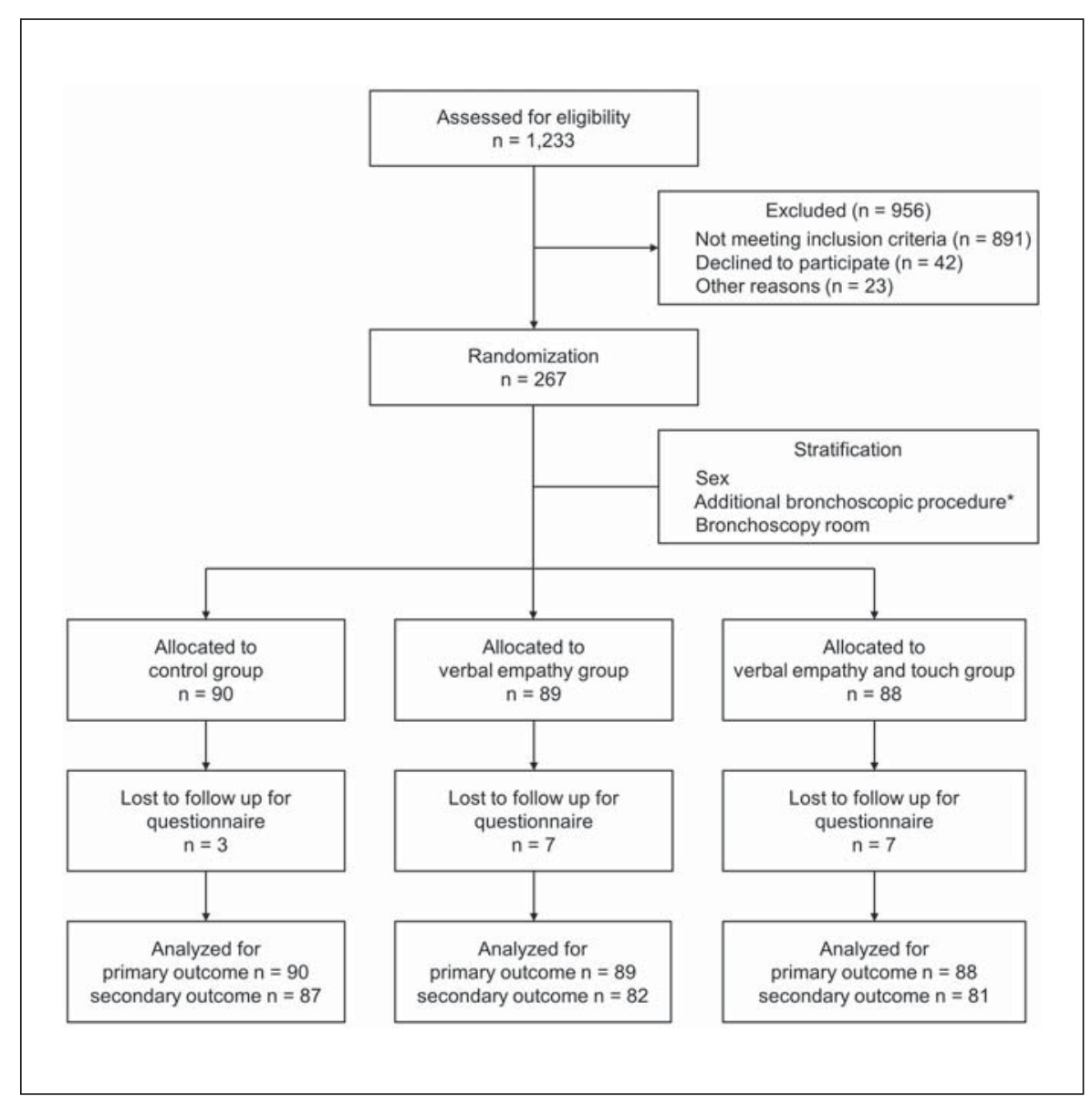

local anesthetics, FB is generally tolerable for patients under conscious sedation $[3,5,6]$. However, like other invasive procedures, bronchoscopy makes patients anxious [7].

Previous attempts to relieve patients' anxiety and improve their satisfaction by the use of music/sound or art during the procedure have been ineffective $[8,9]$. A systematic review reported that music reduced anxiety in patients during normal care delivery, but it had no impact on the anxiety in patients undergoing procedures or surgery [10]. One study reported that distraction therapy with nature sights and sounds provided before, during, and after FB did not reduce anxiety in patients undergoing the procedure, while it significantly reduced pain in the same population [8]. Another study also described that music during FB did not decrease procedure-related anxiety in the patients [9].

Many literatures described that empathy and physical contacts such as hand-holding or touch can enhance the satisfaction of patients, physician-patient communica- tion, quality of care, and even health outcomes [11-13]. There is evidence that these interventions can reduce anxiety in patients undergoing procedures or surgery [14-16]. However, there is no study evaluating the effectiveness of empathy and touch on the level of anxiety in patients undergoing FB.

In this context, we performed this study to investigate whether bronchoscopists' verbal empathy and intentional touch could reduce anxiety in patients undergoing FB.

\section{Materials and Methods}

\section{Participants}

Patients aged 19 years or more undergoing FB in a universityaffiliated hospital in South Korea between November 19, 2013 and September 12, 2014 were eligible for this study. Patients were excluded if they were undergoing endobronchial ultrasound or interventional bronchoscopy, admitted to the intensive care unit, or unable to communicate. Patients for whom bronchoscopy was performed in an outpatient setting and patients who had experi- 


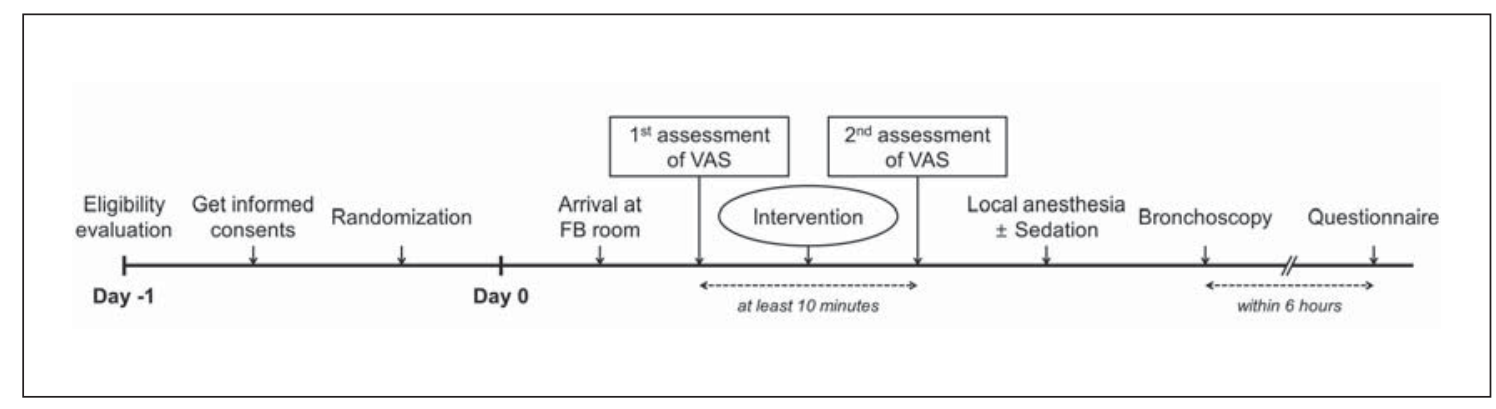

Fig. 2. Phases of the study.

enced bronchoscopy were previously also excluded from the study. All patients provided informed written consent, and the protocol was approved by the Institutional Review Board of Seoul National University Hospital (IRB No. H-1307-042-504) for the study period between September 25, 2013 and September 24, 2014. This trial was registered with clinicaltrials.gov (No. NCT02000245).

\section{Study Design}

The flow diagram and the phases of this study are shown schematically in figures 1 and 2, respectively. A prospective randomized trial including 3 arms was performed. Randomization sequence was created using SAS 9.1 software (SAS Institute, Inc., Cary, N.C., USA) and was stratified based on sex, on whether an additional diagnostic procedure during bronchoscopy (such as bronchoscopic biopsy) was expected to be performed or not, and on the bronchoscopy rooms (we have two bronchoscopy rooms in which different personnel work) by Medical Research Collaborating Center of Seoul National University Hospital with a 1:1:1 allocation using random block sizes of 3 and 6 . The allocations were contained in opaque, sequentially numbered sealed envelopes. Participants were randomly assigned to one of the following groups: a control group, a verbal empathy group, or a verbal empathy and touch group by a nurse who was not involved in the study.

To express verbal empathy, all staff pulmonologists used the following memorized script to communicate (in Korean) with the patient, making eye contact, after patients had checked the baseline level of anxiety. We used a visual analog scale (VAS) to measure the level of patients' anxiety based on the previous studies evaluating anxiety of patients $[9,15,17]$. The results of the VAS were assessed by a technician who was blinded to group assignment.

'Hello, I am Dr. Jane Doe, a staff pulmonologist performing bronchoscopy today. You may feel anxious now. You might feel uncomfortable during the exam, but we are here with you, keeping an eye on you the whole time. Don't worry and relax please.'

(1) Control group: the staff pulmonologist did not say anything to the patient and did not touch him/her before the performance of bronchoscopy.

(2) Verbal empathy group: the staff pulmonologist used the script to express empathy with the patient, making eye contact but avoiding touch.

(3) Verbal empathy and touch group: the staff pulmonologist used the script to express empathy with the patient, simultaneously laying his/her left hand on the patient's right shoulder.

\section{Bronchoscopy Procedure}

Bronchoscopy was performed by a staff pulmonologist or by a pulmonary fellow under the close supervision of a staff pulmonologist in one of the two bronchoscopy rooms.

At least two well-trained nurses or technicians assisted the bronchoscopist during each procedure. The depth of sedation was assessed with the Modified Observer's Assessment of Alertness/ Sedation Scale during the procedure [18]. For local anesthesia, aerosolized lidocaine $(2 \%, 20 \mathrm{ml})$ was sprayed into the oropharynx before insertion of the bronchoscope, and lidocaine solution (1\%, 5-10 ml) was instilled through the bronchoscope after advancing the bronchoscope through the vocal cords. Conscious sedation was induced using midazolam for patients who wanted to be sedated during bronchoscopy. Vital signs were monitored and procedure time, additional procedures, and drugs used during the bronchoscopy were all recorded.

\section{Outcomes}

The primary outcome was the difference in the extent of anxiety change (as measured by the VAS) between the control and verbal empathy groups, and between the control group and the verbal empathy and touch group. The VAS consisted of a $10-\mathrm{cm}$ horizontal line with 0 at the left end (representing no anxiety) and 10 at the right end (representing extreme anxiety). Patients were asked to indicate their current level of anxiety by marking the line. Baseline anxiety was assessed when patients arrived in the bronchoscopy room by a technician blinded to the randomized allocation. The second anxiety assessment took place immediately before the bronchoscopy and was performed by the same personnel. The technician assessing anxiety was not involved in the study and the minimum time interval between the two VAS assessments was $10 \mathrm{~min}$ (fig. 2). Post hoc subgroup analysis including patients with higher baseline anxiety $(\geq 50, \geq 60$, and $\geq 70$ $\mathrm{mm}$ ) was performed.

The secondary outcome was patients' satisfaction after the bronchoscopy. This was evaluated with a questionnaire that participants were asked to complete within $6 \mathrm{~h}$ of the bronchoscopy by a nurse blinded to the randomized allocation. The questionnaire evaluated patients' religion, level of education, comorbidities, and satisfaction (e.g., if the patient was willing to repeat the bronchoscopy if necessary). Patients' discomfort, such as pain, cough, and difficulty breathing during the bronchoscopy, was evaluated. A 5-point scale (definitely not, probably not, unsure, probably would, and definitely would) was used for most of the questions. 
Table 1. Baseline characteristics of patients

\begin{tabular}{|c|c|c|c|c|}
\hline & $\begin{array}{l}\text { Control group } \\
(\mathrm{n}=90)\end{array}$ & $\begin{array}{l}\text { Verbal empathy } \\
\text { group }(n=89)\end{array}$ & $\begin{array}{l}\text { Verbal empathy } \\
\text { and touch group } \\
(\mathrm{n}=88)\end{array}$ & $\mathrm{p}$ value \\
\hline Median age, years (IQR) & $65(55-72)$ & $65(58-72)$ & $66(57-72)$ & 0.962 \\
\hline Male & $56(62.2)$ & $56(62.9)$ & $54(61.4)$ & 0.977 \\
\hline Body mass index & $22.7 \pm 3.1$ & $22.2 \pm 3.4$ & $22.4 \pm 3.2$ & 0.498 \\
\hline Religion & & & & 0.425 \\
\hline Christianity & $23(26.1)$ & $21(25.6)$ & $22(27.5)$ & \\
\hline Catholicism & $7(8.0)$ & $12(14.6)$ & $9(11.3)$ & \\
\hline Buddhism & $12(13.6)$ & $17(20.7)$ & $16(20.0)$ & \\
\hline None & $42(47.7)$ & $32(39.0)$ & $32(40.0)$ & \\
\hline Others & $4(4.5)$ & $0(0.0)$ & $1(1.3)$ & \\
\hline Education & & & & 0.714 \\
\hline College & $26(29.5)$ & $22(26.8)$ & $28(35.0)$ & \\
\hline High school & $29(33.0)$ & $29(35.4)$ & $26(32.5)$ & \\
\hline Middle school & $11(12.5)$ & $12(14.6)$ & $5(6.3)$ & \\
\hline Elementary school & $5(5.7)$ & $2(2.4)$ & $5(6.3)$ & \\
\hline Others & $17(19.3)$ & $17(20.7)$ & $16(20.0)$ & \\
\hline \multicolumn{5}{|l|}{ Comorbidity } \\
\hline Hypertension & $30(34.1)$ & $18(22.0)$ & $14(17.5)$ & 0.035 \\
\hline Diabetes mellitus & $14(16.1)$ & $16(19.5)$ & $13(16.3)$ & 0.806 \\
\hline Liver disease & $0(0.0)$ & $1(1.2)$ & $3(3.8)$ & 0.078 \\
\hline Stroke & $0(0.0)$ & $3(3.7)$ & $0(0.0)$ & 0.066 \\
\hline Depression & $1(1.1)$ & $0(0.0)$ & $0(0.0)$ & 1.000 \\
\hline Insomnia & $0(0.0)$ & $3(3.7)$ & $1(1.3)$ & 0.123 \\
\hline Tumor & $14(15.9)$ & $14(17.1)$ & $17(21.3)$ & 0.644 \\
\hline Asthma & $3(3.5)$ & $0(0.0)$ & $4(5.0)$ & 0.116 \\
\hline Chronic obstructive lung disease & $2(2.3)$ & $2(2.4)$ & $2(2.5)$ & 1.000 \\
\hline Bronchiectasis & $3(3.4)$ & $1(1.2)$ & $2(2.5)$ & 0.783 \\
\hline Tuberculosis & $9(10.2)$ & $3(3.7)$ & $10(12.5)$ & 0.117 \\
\hline \multicolumn{5}{|l|}{ Respiratory symptom } \\
\hline Cough & $36(40.9)$ & $32(39.0)$ & $34(42.5)$ & 0.903 \\
\hline Sputum & $32(36.4)$ & $25(30.5)$ & $30(37.5)$ & 0.599 \\
\hline Dyspnea & $23(26.1)$ & $20(24.4)$ & $21(26.3)$ & 0.954 \\
\hline Hemoptysis & $17(19.3)$ & $7(8.5)$ & $11(13.8)$ & 0.128 \\
\hline Indication of $\mathrm{FB}$ & & & & 0.633 \\
\hline Suspicion of lung cancer & $25(27.8)$ & $32(36.0)$ & $29(33.0)$ & \\
\hline Preoperative evaluation & $19(21.1)$ & $17(19.1)$ & $18(20.5)$ & \\
\hline To identify infectious organisms & $35(38.9)$ & $27(30.3)$ & $27(30.7)$ & \\
\hline Hemoptysis & $6(6.7)$ & $5(5.6)$ & $4(4.5)$ & \\
\hline Interstitial lung disease & $2(2.2)$ & $2(2.2)$ & $7(8.0)$ & \\
\hline Others $^{1}$ & $3(3.3)$ & $6(6.7)$ & $3(3.4)$ & \\
\hline Median level of anxiety on the VAS (IQR) & $37(20-59)$ & $30(10-55)$ & $30(10-60)$ & 0.682 \\
\hline
\end{tabular}

Data are presented as $\mathrm{n}(\%)$ or mean \pm standard deviation unless otherwise indicated. IQR $=$ Interquartile range. ${ }^{1}$ Atelectasis and chronic cough.

\section{Sample Size Determination}

A previous study assessing peribronchoscopy anxiety and satisfaction defined a change of $8 \mathrm{~mm}$ on the VAS as clinically significant, assuming that a clinically significant change in a healthrelated quality of life tool is equivalent to $8 \%$ of the theoretical range of the tool [17]. Sample size calculations were based on the primary outcome, the difference in the VAS change, which should be $8 \mathrm{~mm}$ between the intervention and control groups. The pooled standard deviation was defined as 16.77 based on a previous study assessing changes in pain VAS [19]. A power calculation suggested that a total sample size of 267 patients would have $80 \%$ power to detect an 8-mm difference in VAS score change between the intervention groups and the control group at a significance level of $\mathrm{p}<$ $0.025(=0.05 / 2)$. 
Table 2. Procedure-related variables

\begin{tabular}{|c|c|c|c|c|}
\hline & $\begin{array}{l}\text { Control group } \\
(\mathrm{n}=90)\end{array}$ & $\begin{array}{l}\text { Verbal empathy } \\
\text { group }(n=89)\end{array}$ & $\begin{array}{l}\text { Verbal empathy } \\
\text { and touch group } \\
(\mathrm{n}=88)\end{array}$ & $\mathrm{p}$ value \\
\hline Male bronchoscopist & $57(63.3)$ & $55(61.8)$ & $52(59.1)$ & 0.841 \\
\hline \multicolumn{5}{|l|}{ Procedure } \\
\hline Washing & $54(61.4)$ & $54(60.7)$ & $54(62.8)$ & 0.958 \\
\hline Biopsy & $23(25.6)$ & $22(24.7)$ & $17(19.5)$ & 0.593 \\
\hline Bronchoalveolar lavage & $18(20.0)$ & $12(13.5)$ & $17(19.5)$ & 0.445 \\
\hline Transbronchial lung biopsy & $1(1.1)$ & $2(2.2)$ & $0(0.0)$ & 0.660 \\
\hline Brushing & $3(3.3)$ & $1(1.1)$ & $0(0.0)$ & 0.328 \\
\hline Oral insertion & $88(97.8)$ & $87(97.8)$ & $85(98.8)$ & $>0.999$ \\
\hline Procedure duration, min & $10.8 \pm 5.9$ & $11.1 \pm 7.7$ & $10.1 \pm 6.3$ & 0.573 \\
\hline \multicolumn{5}{|l|}{ Vital signs (before FB) } \\
\hline Systolic blood pressure & $127.2 \pm 20.5$ & $131.4 \pm 22.2$ & $128.6 \pm 19.0$ & 0.385 \\
\hline Diastolic blood pressure & $72.7 \pm 10.1$ & $75.7 \pm 11.7$ & $74.5 \pm 10.8$ & 0.196 \\
\hline Heart rate & $76.8 \pm 14.0$ & $77.6 \pm 14.8$ & $78.4 \pm 18.0$ & 0.783 \\
\hline Respiratory rate & $17.7 \pm 2.7$ & $17.5 \pm 2.1$ & $17.3 \pm 2.5$ & 0.496 \\
\hline Oxygen saturation & $97.2 \pm 2.5$ & $97.5 \pm 2.3$ & $97.5 \pm 2.2$ & 0.523 \\
\hline \multicolumn{5}{|l|}{ Vital signs (after FB) } \\
\hline Systolic blood pressure & $135.7 \pm 24.5$ & $142.2 \pm 23.6$ & $141.1 \pm 22.6$ & 0.151 \\
\hline Diastolic blood pressure & $77.8 \pm 11.2$ & $82.2 \pm 12.9$ & $82.3 \pm 12.5$ & 0.023 \\
\hline Heart rate & $91.1 \pm 14.7$ & $89.4 \pm 17.1$ & $90.5 \pm 18.1$ & 0.787 \\
\hline Respiratory rate & $20.8 \pm 3.1$ & $20.4 \pm 2.7$ & $20.0 \pm 3.3$ & 0.302 \\
\hline Oxygen saturation & $96.5 \pm 2.4$ & $96.9 \pm 2.3$ & $97.0 \pm 2.4$ & 0.306 \\
\hline \multicolumn{5}{|l|}{ Drugs } \\
\hline Midazolam, mg & $4.4 \pm 2.2$ & $4.3 \pm 1.8$ & $4.5 \pm 1.8$ & 0.833 \\
\hline Lidocaine $^{1}, \mathrm{mg}$ & $273 \pm 152$ & $251 \pm 125$ & $238 \pm 105$ & 0.183 \\
\hline Flumazenil & $43(64.2)$ & $34(57.6)$ & $42(61.8)$ & 0.750 \\
\hline Median MOAA/S score (IQR) & $4(1-5)$ & $3(1-5)$ & $3(1-5)$ & 0.332 \\
\hline
\end{tabular}

Data are presented as $\mathrm{n}(\%)$ or mean \pm standard deviation unless otherwise indicated. IQR $=$ Interquartile range. ${ }^{1}$ Intrabronchial.

Statistical Analysis

The $\chi^{2}$ test or Fisher's exact test were used to compare categorical variables and the Mann-Whitney $U$ test for continuous variables. A p value of $<0.05$ was considered statistically significant. SPSS 19.0 statistical software (IBM Corp., Armonk, N.Y., USA) was used for analysis. Subgroup analysis was performed to evaluate the effect of baseline level of anxiety of the participants on the outcome.

\section{Results}

\section{Baseline Characteristics of the Participants}

A total of 267 patients were enrolled in this study. The median age was 65 years and $62.2 \%$ were men. There were no statistically significant differences in age, sex, body mass index, religion, level of education, and comorbidities between the three groups, except that hypertension was more common in the control group. The most common respiratory symptoms were cough (40.8\%) and sputum (34.8\%). The main indications of bronchoscopy were to identify infectious organisms (33.3\%) and suspicion of lung cancer (31.8\%) (table 1). The baseline levels of anxiety before bronchoscopy were not different between groups of patients with different indications.

\section{Procedure-Related Variables}

A total of 209 (78.3\%) patients underwent at least one bronchoscopic procedure including bronchial washing, endobronchial biopsy, bronchoalveolar lavage, transbronchial lung biopsy, and brushing. There were no differences in the type of procedure, insertion route (nose vs. mouth), and procedure duration. The amounts of instilled lidocaine and intravenously injected midazolam were also similar. The median sedation score on the Modified Observer's Assessment of Alertness/Sedation Scale 
Table 3. Changes in anxiety VAS assessment

$\begin{array}{llll}\begin{array}{l}\text { Control group } \\ (\mathrm{n}=90)\end{array} & \begin{array}{l}\text { Verbal empathy } \\ \text { group }(\mathrm{n}=89)\end{array} & \begin{array}{l}\text { Verbal empathy } \\ \text { and touch group } \\ (\mathrm{n}=88)\end{array} & \begin{array}{l}\text { p value } \\ \text { control vs. control vs. } \\ \text { empathy empathy } \\ \text { and touch }\end{array}\end{array}$

\begin{tabular}{|c|c|c|c|c|c|}
\hline \multicolumn{6}{|l|}{ Change in VAS level } \\
\hline Total & $-0.31(-2.9,2.3)$ & $-1.17(-4.1,1.8)$ & $-1.89(-5.7,1.9)$ & 0.285 & 0.431 \\
\hline Patients with baseline VAS $\geq 50 \mathrm{~mm}^{*}$ & $-5.29(-9.5,-1.1)$ & $-6.10(-12.0,-0.2)$ & $-12.05(-19.0,-5.1)$ & 0.526 & 0.153 \\
\hline Patients with baseline VAS $\geq 60 \mathrm{~mm}^{\dagger}$ & $-4.95(-10.8,0.9)$ & $-10.05(-17.0,-3.1)$ & $-16.20(-24.8,-7.6)$ & 0.157 & 0.039 \\
\hline Patients with baseline VAS $\geq 70 \mathrm{~mm}^{\ddagger}$ & $-1.33(-7.1,4.4)$ & $-14.08(-22.1,-6.0)$ & $-18.53(-28.1,-8.9)$ & 0.011 & 0.003 \\
\hline \multicolumn{6}{|l|}{ Patients with decreased VAS level } \\
\hline Total & $34(37.8)$ & $47(52.8)$ & $38(43.2)$ & 0.043 & 0.463 \\
\hline Baseline VAS $\geq 50 \mathrm{~mm}^{*}$ & $18(52.9)$ & $20(66.7)$ & $19(63.3)$ & 0.265 & 0.401 \\
\hline Baseline VAS $\geq 60 \mathrm{~mm}^{\dagger}$ & $9(40.9)$ & $15(75.0)$ & $16(72.7)$ & 0.026 & 0.033 \\
\hline Baseline VAS $\geq 70 \mathrm{~mm}^{\ddagger}$ & $4(26.7)$ & $11(84.6)$ & $14(77.8)$ & 0.002 & 0.003 \\
\hline \multicolumn{6}{|l|}{ Patients with decreased VAS level $\geq$ MCID } \\
\hline Total & $22(24.4)$ & $25(28.1)$ & $24(27.3)$ & 0.579 & 0.667 \\
\hline Baseline VAS $\geq 50 \mathrm{~mm}^{*}$ & $13(38.2)$ & $14(46.7)$ & $15(50.0)$ & 0.496 & 0.344 \\
\hline Baseline VAS $\geq 60 \mathrm{~mm}^{\dagger}$ & $8(36.4)$ & $11(55.0)$ & $13(59.1)$ & 0.226 & 0.131 \\
\hline Baseline VAS $\geq 70 \mathrm{~mm}^{\ddagger}$ & $4(26.7)$ & $9(69.2)$ & $11(61.1)$ & 0.024 & 0.048 \\
\hline
\end{tabular}

Data are presented by $\mathrm{n}(\%)$ or mean (95\% confidence intervals) unless otherwise indicated. MCID = Minimal clinically important difference $(=8 \mathrm{~mm}){ }^{*} \mathrm{n}=94($ control group $=34$, verbal empathy group $=30$, verbal empathy and touch group $=30) ;{ }^{\dagger} \mathrm{n}=64($ control group $=22$, verbal empathy group $=20$, verbal empathy and touch group $=22) ;{ }^{\ddagger} n=46($ control group $=15$, verbal empathy group $=$ 13 , verbal empathy and touch group $=18$ ).

was similar for the control, empathy, and empathy and touch groups $(4,3$, and 3 , respectively, $\mathrm{p}=0.332)$. Preand post-FB vital signs were similar, but the control group showed lower post-FB diastolic blood pressure $(\mathrm{p}=0.023)$ (table 2).

\section{Anxiety Change Measured with the VAS}

There was no difference in anxiety VAS change (the primary outcome) between the control group and the verbal empathy group ( -0.31 vs. $-1.17, \mathrm{p}=0.285)$, and between the control group and the verbal empathy and touch group $(-0.31$ vs. $-1.89, \mathrm{p}=0.431)$. Subgroup analysis including patients with baseline anxiety VAS $\geq 60 \mathrm{~mm}$ showed a greater decrease in anxiety in the empathy and touch group compared with the control group ( $-16.20 \mathrm{vs.}$ $-4.95, \mathrm{p}=0.039$ ), although no statistical difference was found between the empathy group and the control group $(-10.05$ vs. $-4.95, \mathrm{p}=0.157)$. Among those with baseline anxiety VAS $\geq 70 \mathrm{~mm}$, the empathy and touch group $(-18.53)$ and the empathy group $(-14.08)$ showed a significant decrease in anxiety compared with the control group $(-1.33, \mathrm{p}=0.003$ and $\mathrm{p}=0.011$, respectively) (table 3$)$.

Verbal Empathy and Touch and Anxiety

Relief in Patients Undergoing FB

\section{Satisfaction and Symptoms Related to Bronchoscopy}

Overall participant satisfaction was good and not significantly different between the three groups. Of those patients who indicated that they would be willing to return if necessary (including definitely yes and probably yes responses), 73 (83\%) were in the control group, 71 (86.5\%) in the empathy group, and $65(81.3 \%)$ in the empathy and touch group $(\mathrm{p}=0.196)$. There was no difference in satisfaction with prebronchoscopy information, feeling uncomfortable, pain, and cough during bronchoscopy between the three groups (table 4).

\section{Discussion}

FB is one of the most important procedures to identify various pathologic conditions in respiratory medicine. However, the procedure can cause patients' anxiety [7] because it is performed through the airway and may be accompanied by invasive procedures such as transbronchial lung biopsy. Addressing patients' anxiety is important because anxiety affects the tolerability and performance of the procedure, influences patients' satisfaction 
Table 4. Patient satisfaction

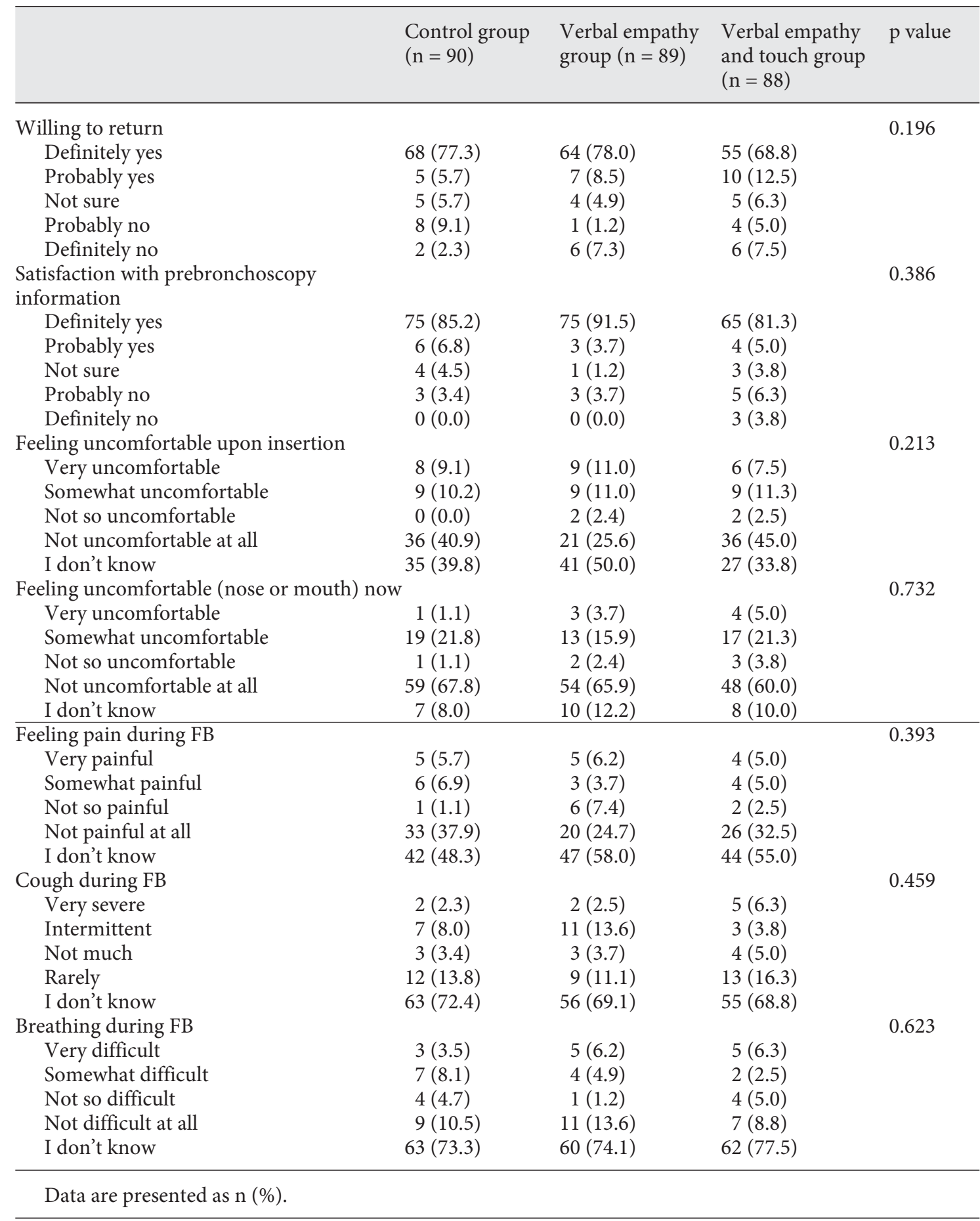

in the quality of care, and might result in reduced compliance with medical treatment [20-22]. Previous studies using natural sights and sounds [8] or music [9] have failed to reduce the anxiety in patients undergoing FB.
Our findings here indicate that a verbal expression of empathy accompanied by the bronchoscopist's touch decreased anxiety among those patients undergoing bronchoscopy with higher baseline anxiety VAS scores. 
Patients regard empathy as a key component of the therapeutic relationship and quality of care [23]. Empathy is also a basic constituent of a good doctor-patient relationship, which improves the quality of care and treatment outcomes $[12,13]$ and helps relieve patient anxiety. One previous study reported that patients who perceived their physician as empathic reported lower levels of anxiety [24]. In another randomized controlled trial, a standardized videotape intervention featuring enhanced physician compassion significantly decreased viewers' anxiety [14].

Physical contact is also effective in reducing patients' anxiety and improving their emotional state. In one previous study, hand-holding during cataract surgery under local anesthesia was found to reduce patients' anxiety [15], and several nursing studies have reported that provision of intentional touch empowered patients and had positive effects on their emotions and feelings $[25,26]$.

In this study, the anxiety-relieving effects of verbal empathy and touch were observed only in participants with higher baseline anxiety levels. There was an effect of verbal empathy accompanied by intentional touch in participants with baseline anxiety on the VAS of $\geq 60 \mathrm{~mm}$, and verbal empathy with touch as well as verbal empathy without touch reduced anxiety in participants with baseline anxiety on the VAS of $\geq 70 \mathrm{~mm}$. This observation can be understood in the context of previous studies suggesting that patients with higher preintervention anxiety lev- els are more vulnerable to the aggravation of anxiety, or more susceptible to anxiety relief by intervention [17, $27,28]$.

The lack of an effect for verbal empathy and touch among all the participants may be explained by the study's limitations. First, the form of verbal expression we used, lasting about $25 \mathrm{~s}$, may have been too short to reveal physicians' empathy. In a previous study that reported positive results of verbal empathy, the duration of expression of enhanced compassion was approximately $40 \mathrm{~s}$ [14]. In addition, our verbal empathy was a one-way expression rather than interaction with participants. The intensity and duration of touch may also not have been sufficient to indicate physicians' empathy. Physicians simply placed a hand on the patient's shoulder while expressing verbal empathy, which took approximately $25 \mathrm{~s}$. In one previous study that reported a positive effect of hand-holding on patients' anxiety, the nurse practitioner held the patient's hand throughout the surgery [15]. Second, the bronchoscopist providing verbal empathy and touch was not the participants' duty physician in the majority of the cases. In those cases, participants had met the bronchoscopist for the first time; this could have been a barrier to showing empathy.

In conclusion, verbal empathy and touch provided before bronchoscopy reduced anxiety in patients with high baseline anxiety levels. Further research is needed to test other interventions to relieve patients' anxiety while undergoing bronchoscopy or other medical procedures.

\section{References}

$>1$ Pittman S, Kridli S: Music intervention and preoperative anxiety: an integrative review. Int Nurs Rev 2011;58:157-163.

$>2$ Huber JD, Pukall CF, Boyer SC, et al: 'Just relax': physicians' experiences with women who are difficult or impossible to examine gynecologically. J Sex Med 2009;6:791-799.

$\checkmark 3$ Putinati S, Ballerin L, Corbetta L, et al: Patient satisfaction with conscious sedation for bronchoscopy. Chest 1999;115:1437-1440.

4 Mahmood K, Wahidi MM, Thomas S, et al: Therapeutic bronchoscopy improves spirometry, quality of life, and survival in central airway obstruction. Respiration 2015;89:404413.

5 Wahidi MM, Jain P, Jantz M, et al: American College of Chest Physicians consensus statement on the use of topical anesthesia, analgesia, and sedation during flexible bronchoscopy in adult patients. Chest 2011;140:13421350 .

Verbal Empathy and Touch and Anxiety

Relief in Patients Undergoing FB
-6 Maguire GP, Rubinfeld AR, Trembath PW, et al: Patients prefer sedation for fibreoptic bronchoscopy. Respirology 1998;3:81-85.

$>7$ Poi PJ, Chuah SY, Srinivas P, et al: Common fears of patients undergoing bronchoscopy. Eur Respir J 1998;11:1147-1149.

-8 Diette GB, Lechtzin N, Haponik E, et al: Distraction therapy with nature sights and sounds reduces pain during flexible bronchoscopy: a complementary approach to routine analgesia. Chest 2003;123:941-948.

$\checkmark$ Colt HG, Powers A, Shanks TG: Effect of music on state anxiety scores in patients undergoing fiberoptic bronchoscopy. Chest 1999; 116:819-824.

10 Evans D: The effectiveness of music as an intervention for hospital patients: a systematic review. J Adv Nurs 2002;37:8-18.
1 Bruhn JG: The doctor's touch: tactile communication in the doctor-patient relationship. South Med J 1978;71:1469-1473.

12 Stewart MA: Effective physician-patient communication and health outcomes: a review. CMAJ 1995; 152:1423-1433.

13 Derksen F, Bensing J, Lagro-Janssen A: Effectiveness of empathy in general practice: a systematic review. Br J Gen Pract 2013;63:e76-

e84.
14 Fogarty LA, Curbow BA, Wingard JR, et al: Can 40 seconds of compassion reduce patient anxiety? J Clin Oncol 1999;17:371-379.

15 Moon JS, Cho KS: The effects of handholding on anxiety in cataract surgery patients under local anaesthesia. J Adv Nurs 2001;35:407415.

16 Kim BH, Kang HY, Choi EY: Effects of handholding and providing information on anxiety in patients undergoing percutaneous vertebroplasty. J Clin Nurs 2015;24:3459-3468. 
17 Uzbeck M, Quinn C, Saleem I, et al: Randomised controlled trial of the effect of standard and detailed risk disclosure prior to bronchoscopy on peri-procedure anxiety and satisfaction. Thorax 2009;64:224-227.

18 Chernik DA, Gillings D, Laine H, et al: Validity and reliability of the Observer's Assessment of Alertness/Sedation Scale: study with intravenous midazolam. J Clin Psychopharmacol 1990;10:244-251.

19 Tubach F, Ravaud P, Baron G, et al: Evaluation of clinically relevant changes in patient reported outcomes in knee and hip osteoarthritis: the minimal clinically important improvement. Ann Rheum Dis 2005;64:29-33.

20 Yunusa MA, Njoku CH, Obembe A: Self-reported adherence to treatment: a study of socioeconomic factors and psychiatric morbidity among male and female patients with HIV infection in Sokoto, Nigeria. Running title: Treatment adherence, socioeconomic factors and psychiatric morbidity in HIV patients. Niger J Med 2014;23:33-39.

-21 Alcantara C, Edmondson D, Moise N, et al: Anxiety sensitivity and medication nonadherence in patients with uncontrolled hypertension. J Psychosom Res 2014;77:283-286.

22 Sundbom LT, Bingefors K: The influence of symptoms of anxiety and depression on medication nonadherence and its causes: a population based survey of prescription drug users in Sweden. Patient Prefer Adherence 2013;7: 805-811.

23 Mercer SW, Reynolds WJ: Empathy and quality of care. Br J Gen Pract 2002;52(suppl):S9S12.

24 van Dulmen S, van den Brink-Muinen A: Patients' preferences and experiences in han- dling emotions: a study on communication sequences in primary care medical visits. $\mathrm{Pa}$ tient Educ Couns 2004;55:149-152.

-25 Edvardsson JD, Sandman PO, Rasmussen $\mathrm{BH}$ : Meanings of giving touch in the care of older patients: becoming a valuable person and professional. J Clin Nurs 2003;12:601609.

26 Butts JB: Outcomes of comfort touch in institutionalized elderly female residents. Geriatr Nurs 2001;22:180-184.

27 Kerrigan DD, Thevasagayam RS, Woods TO, et al: Who's afraid of informed consent? BMJ 1993;306:298-300.

28 Granziera E, Guglieri I, Del Bianco P, et al: A multidisciplinary approach to improve preoperative understanding and reduce anxiety: a randomised study. Eur J Anaesthesiol 2013; 30:734-742. 